\title{
Slow dynamics in the three-dimensional gonihedric model
}

\author{
P. Dimopoulos, ${ }^{*}$ D. Espriu, ${ }^{\dagger}$ E. Jané,${ }^{\ddagger}$ and A. Prats ${ }^{\S}$ \\ Departament d'Estructura i Constituents de la Materia, Universitat de Barcelona, Diagonal 647, 08028 Barcelona, Spain
}

(Received 25 April 2002; revised manuscript received 27 June 2002; published 19 November 2002)

\begin{abstract}
We study dynamical aspects of three-dimensional gonihedric spins by using Monte-Carlo methods. These models have a purely geometrical motivation, deriving from string and random surface theory. Here, however, we shall analyze this family of models just from a statistical point of view. In particular, we shall be concerned with their ability to exhibit remarkably slow dynamics and seemingly glassy behavior below a certain temperature $T_{g}$, without the need of introducing disorder of any kind. We consider first a Hamiltonian that takes into account only a four-spin term $(\kappa=0)$, where a first-order phase transition is well established. By studying the relaxation properties at low temperatures, we confirm that the model exhibits two distinct regimes. For $T_{g}<T<T_{c}$, with long lived metastability and a supercooled phase, the approach to equilibrium is well described by a stretched exponential. For $T<T_{g}$, the dynamics appears to be logarithmic. We provide an accurate determination of $T_{g}$. We also determine the evolution of particularly long lived configurations. Next, we consider the case $\kappa=1$, where the plaquette term is absent and the gonihedric action consists in a ferromagnetic Ising with fine-tuned next-to-nearest neighbor interactions. This model exhibits a second order phase transition. The consideration of the relaxation time for configurations in the cold phase reveals the presence of slow dynamics and glassy behavior for any $T<T_{c}$. Type-II aging features are exhibited by this model.
\end{abstract}

DOI: 10.1103/PhysRevE.66.056112 PACS number(s): 05.50.+q, 75.40.Cx, 75.40.Gb, 75.70.Kw

\section{INTRODUCTION}

Glassy systems are very common in nature, yet not quite well understood. Lattice models may serve as good candidates to describe some properties of these systems. In recent years some interest has been raised by remarkably simple Ising spin systems that originated from the consideration of a model of random surfaces in the context of string theory $[1,2]$. The version of this model in a discretized space defines the so-called gonihedric spin system, which consists of an Ising model with finely tuned nearest, next-to-nearest neighbor, and plaquette interactions. The relation among the couplings of the Hamiltonian depends also on the dimensionality of the system. The geometric origins of the model show up in a remarkable simple way of writing the energy of a given configuration: the surfaces corresponding to the interfaces between up and down spins are weighed by $E=n_{2}+4 \kappa n_{4}$, where $n_{2}$ is the number of edges of such an interface and $n_{4}$ is the number of four plaquettes that share a common link in the dual lattice. The parameter $\kappa$ can thus be interpreted as an indicator of the self-avoidance of the model. Notice that the main peculiarity of this family of models (parametrized by $\kappa$ ) is that the microscopic surface tension vanishes. Fluctuations do generate a macroscopic string tension, however [3].

Originally, the interest of the model lies in its relation to the string and random surface theory. Soon it was realized that the model was, in addition, rather interesting in itself. It is, in fact, a special case of a very general family of models containing nearest, next-to-nearest, and plaquette interactions

\footnotetext{
*Email address: petros@ecm.ub.es

${ }^{\dagger}$ Email address: espriu@ecm.ub.es

†Email address: ejane@ecm.ub.es

${ }^{\S}$ Email address: prats@ecm.ub.es
}

which have been studied in detail in the past. However, gonihedric models correspond to a corner in parameter space of the above models where the usual analysis does not apply, perhaps because the model possesses-exactly at that corner of parameter space and only there-a rich set of symmetries that makes them rather unique. Early studies found that the critical properties of the model appeared to be nonstandard [3]. Soon afterwards it was realized that the model exhibits slow dynamics and seemingly glassy behavior. We shall dwell more on the specific peculiarities of this family of models later. Let us first see the type of models we are talking about.

For the three-dimensional case that is of our concern in this paper, the Hamiltonian of the model takes the form

$$
\begin{aligned}
H(\sigma)= & -2 \kappa \sum_{\vec{r}, \alpha} \sigma_{r} \sigma_{r}+\vec{\alpha}+\frac{\kappa}{2} \sum_{\vec{r}, \vec{\alpha}, \vec{\beta}} \sigma_{r} \sigma_{r+\vec{\alpha}+\vec{\beta}} \\
& -\frac{1-\kappa}{2} \sum_{\vec{r}, \vec{\alpha}, \vec{\beta}} \sigma_{\vec{r}} \sigma_{\vec{r}+\vec{\alpha}} \sigma_{\vec{r}+\vec{\alpha}+\vec{\beta}} \sigma_{\vec{r}+\vec{\beta}},
\end{aligned}
$$

where $\vec{\alpha}$ and $\vec{\beta}$ are lattice unit vectors. The model is defined on an cubic lattice. The system exhibits a very high degree of symmetry due to the particular ratio of the couplings. This symmetry implies that flipping any plane of spins has no energy cost. This results in a highly degenerate ground state. We note in passing that this last feature appears to be common in glassy systems.

Up to now there has been already a substantial amount of numerical work on the three-dimensional case, ${ }^{1}$ to which we shall refer in the following and also some preliminary results in four-dimensional case [5]. In two dimensions, the model

\footnotetext{
${ }^{1}$ For the interesting case of the four-spin model where randomly distributed couplings are considered, see (Ref. [4]).
} 
with $\kappa=0$ is actually trivial (no phase transition [6]) but the analytic solution with $\kappa \neq 0$ is unknown. In fact, the energy can be written as $E=n_{2}+4 \kappa n_{4}$ because of the specific ratios between the couplings. Although, so far no one has succeeded in solving the model in three dimensions, this system is so "geometric" in nature that some attempts managed to come remarkably close to that (see e.g., Ref. [7]). This fact adds to the interest of the model.

Preliminary results for $\kappa \neq 0$ were presented in Ref. [3] in respect of the equilibrium properties. There it was found that the phase transition turns to second order beyond a certain value of $\kappa$ (not precisely determined yet) which, according to the cluster variational method analysis, is most likely a tricritical proint. This second-order transition holds certain surprises not fully understood at present. First of all, the set of exponents determined by finite size scaling in the only calculation so far [3] appears to be nonstandard. Second it was found that abnormally large relaxation times were present in the system.

This last feature was not totally unexpected, since the system for $\kappa=1$ is contained in the family of Hamiltonians described in Ref. [8] as a very particular limiting case. (In Ref. [8] logarithmically slow domain growth was found.) Due to the special ratio of couplings contained in Eq. (1) many of the considerations presented in Ref. [8] may not apply here. For instance, in the gonihedric model the ground state is not necessarily ferromagnetic; rather it can be any of the infinitely (in the thermodynamic limit) degenerate ground states. Furthermore, in this particular limiting case the slow dynamics is seen to persist all the way up to the thermodynamic transition, while in Ref. [8] only low temperatures were considered.

Let us turn to the case $\kappa=0$ where the model is known to have a first-order transition at temperature $T_{c}$, where the solid to liquid transition is present [9]. ${ }^{2}$ This is in agreement with some theoretical work where the cluster variational method [11] was used. These theoretical analyses also show the singular character of the choice of couplings in Eq. (1), dictated by geometry. Good evidence exists for this particular value of $\kappa$ (where no competing nearest and next-tonearest neighbor interactions are present) that a dynamical transition appears at a temperature $T_{g}<T_{c}$, which seems to mark the onset of the glassy behavior [12]. The existence of $T_{g}$ and the study of the dynamical properties of the system above and below that value of the temperature have been considered in Refs. [13-17]. Although the model is certainly very different from the ones considered in Ref. [8], one is tempted to give a similar explanation for the slow dynamics: in the course of the evolution the system finds itself trapped in potential wells of its own making-i.e., dynamically created-and overcoming such barriers would make the evolution slow in any local evolution algorithm. However, this explanation may be a bit too simplistic: the large amount of symmetry presented in Eq. (1) makes flipping any number of planes costless, making the dynamical evolution rather non-

\footnotetext{
${ }^{2} \mathrm{An}$ anisotropic version of this model and the phase diagram is studied in Ref. [10].
}

obvious. In fact, the actual role played by the infinite degeneration in the slow evolution of the gonihedric model deserves investigation.

In Sec. II we provide a qualitative view of the slow dynamics behavior of the cold phase for the $\kappa=0$ case by considering the relaxation properties where two (of the infinitely many) states of the model come in contact. To this end we place the system in an initial configuration where it is forced to move towards a particular ground state. Our results clearly show slow dynamical behavior, not very different from the one previously obtained when the coarsening process is observed after quenching a hot (random) initial configuration [15]. We are able to analyze the short-time and long-time dynamics. An initial readjustment with fast dynamics is followed by an extremely slow evolution. At low temperatures, even after long Monte Carlo (MC) times, the thermal fluctuations fail to lead the system to the ground state (a fact that could prove useful from a practical point of view). In addition, we are able to estimate with good accuracy $T_{g}$ by measuring the spin-spin autocorrelation functions. We show that there is a dramatic change in the behavior of this function above and below $T_{g}$. The temperature $T_{g}$ coincides with the onset of metastability and long lived supercooled states, a region that extends from $T_{g}$ to the (firstorder) transition $T_{c}$ and where the dynamics turns from being logarithmic to a stretched exponential type.

In Sec. III we study a different case of the spin gonihedric action by taking $\kappa=1$ in Eq. (1), apparently a much simpler system (the plaquette term in the Hamiltonian is absent for this value). For that value of the parameter $\kappa$, the system is described by nearest and next-to-nearest interactions. For such a system the ratio of the couplings between the two types of interaction in the Hamiltonian is of great importance. In Ref. [8] there is an extensive analytical and numerical work concerning the dynamics of the model as the ratio of the two interaction type couplings takes large values and at least greater than 4 in order for the system to have a stable ferromagnetic ground state. Our case lies precisely just outside the domain of applicability of this work as the ratio here is exactly 4 . This is due to the special geometrical character of the gonihedric model and is very much related to the high degeneracy of the ground state. We confirm, by studying the energy susceptibility, that the system has a second-order phase transition. ${ }^{3}$ Furthermore, we find slow dynamics behavior anywhere in the cold phase (below the critical temperature). By studying spin-spin autocorrelation function and the overlap spin distribution function, we provide evidence that the system exhibits type-II aging [19], which is a feature of glassy systems. Our results indicate that for the $\kappa=1$ case the thermodynamical transition temperature coincides with the dynamical one and the whole cold phase defines a region of slow dynamics behavior. Thus, from a purely statistical point of view our work for this value of $\kappa$ extends and complements the one presented in Ref. [8].

\footnotetext{
${ }^{3}$ Note that from some preliminary results referred to in Ref. [9], the first order transition present for $\kappa=0$ gets weaker and possibly becomes second order at $\kappa \sim 0.5$.
} 


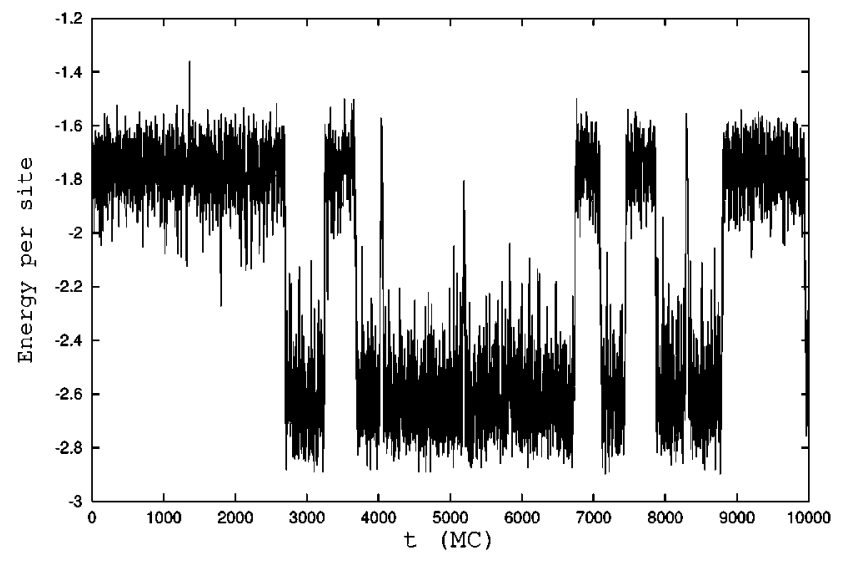

(a)

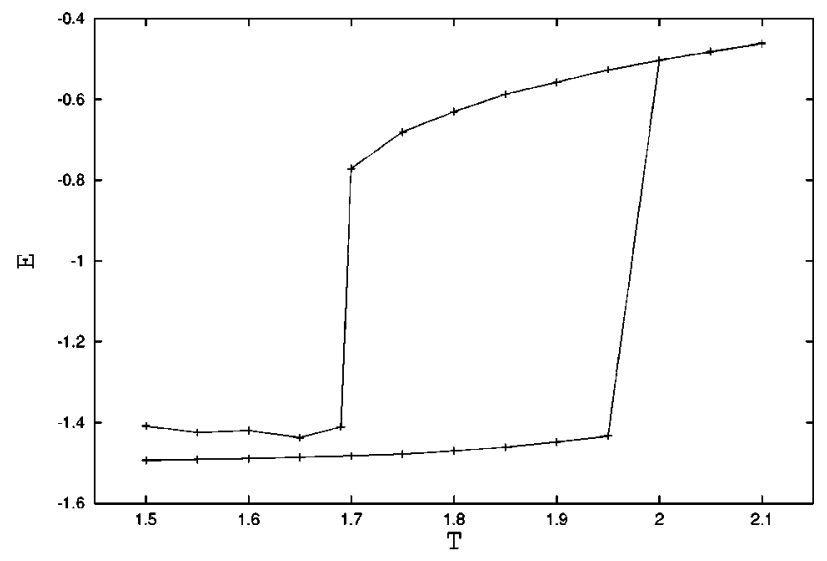

(b)

FIG. 1. (a) An example showing the presence of metastability for $V=8^{3}$; (b) the jumping from the supercooled to the equilibrium phase at $T=1.7$, for $V=24^{3}$.

The physical interest of this model is twofold. On the formal side, the model is of such simplicity that a theoretical understanding of the mechanisms underlying slow dynamics and glassy behavior appears possible. On a more practical side, it would be extremely interesting to be able to understand and produce magnetic materials and coatings with such finely tuned (or approximately so) couplings. The extremely long relaxation times would make them very robust againts thermal noise and fluctuations, yet encoding information there would be as simple as in a normal magnetic material. This possibility has been suggested in Ref. [20]. While the plaquette term seems hard to imitate in real materials, the fact that many of the interesting features persist for $\kappa=1$ makes perhaps such possibility less remote.

\section{FOUR-SPIN INTERACTION $(\kappa=0)$}

In this section we will study the case $\kappa=0$, i.e., a spin model with only four-spin (plaquette) interaction. In this case the Hamiltonian (1) takes the form

$$
H=-\frac{1}{2} \sum_{\vec{r}, \vec{\alpha}, \vec{\beta}} \sigma_{\vec{r}} \sigma_{\vec{r}+\vec{\alpha}} \sigma_{r+\vec{\alpha}+\vec{\beta}} \sigma_{r+\vec{\beta}}
$$

This form of interaction leads to a highly degenerated ground state. Flipping every spin in any plane of the cubic lattice implies invariant ground state energy. Taking this symmetry into account, the degenerancy of the ground state is equal to $2^{3 L}$ due to the $3 L$ diferent planes in a cubic lattice. This degeneracy survives even at $T \neq 0$.

It is well known that this model exhibits a first-order phase transition at $T_{c}=1.95$ along with a dynamical transition at $T_{g} \sim 1.7$, which is the temperature where the glassy behavior shows up [12-14]. Even though our main interest is the study of the glassy characteristics by looking at the relaxation as well as the autocorrelation of the order parameters (to be defined below) in the glassy phase, the region $T_{g}<T<T_{c}$ is interesting as well. In this region, numerical simulations clearly indicate the presence of metastability. This is exemplified by the result presented in Fig. 1(a) where the time evolution of an $8^{3}$ volume at a temperature value just below $T_{c}$ is shown. ${ }^{4}$ This figure also gives clear evidence that there is a first order phase transition. Figure 1(b) consists of two different curves. One of them corresponds to a heating process starting from an initial ordered configuration. The other one describes the result of quenching a random initial configuration for each temperature value shown. In both cases the simulation has been carried out using the METROPOLIS algorithm on a $24^{3}$ volume by performing $10^{4}$ measurements at each temperature value. As we will see below, the approach to equilibrium in the region $T_{g}<T<T_{c}$ is nonstandard and it is well described by a stretched exponential, instead of a simple exponential. For $T<T_{g}$ one immediately sees that the results from the quench of the random configuration after $10^{4} \mathrm{MC}$ steps differ from those obtained starting from an ordered configuration (any of the $2^{3 L}$ vacua) [12]. The difference appears to be constant all over this region (for a fixed number of thermalization steps). This clearly hints at the coexistence of two different dynamics. Initially fast dynamics quickly brings an initial configuration that is badly out of equilibrium to some sort of approximate equilibrium. At that point slow dynamics takes over and the evolution of the system is considerably freezed.

Before getting into the more quantitative aspects of these results, it is perhaps interesting to turn to one of our motivations, namely to test whether the appearence of slow dynamics makes the transition between two approximate ground states so slow as to make a given configuration virtually indestructible by thermal fluctuations, thus providing a convenient way of storing information.

To this end, we simulate the system on a cubic lattice and we use a GLAUBER algorithm [21], which is assumed to provide a good approximation to the thermal mechanism of fluctuations. METROPOLIS or heat bath give very similar results. We look at the decay of an artificial initial configuration consisting in an inner volume with a chessboardlike arrangement of the spins, which is one of the ground states, while the outer volume spins are fixed at +1 , i.e., they form a

\footnotetext{
${ }^{4}$ This simulation refers to the system expressed in dual variables [22] and it has been carried out using a cluster algorithm.
} 


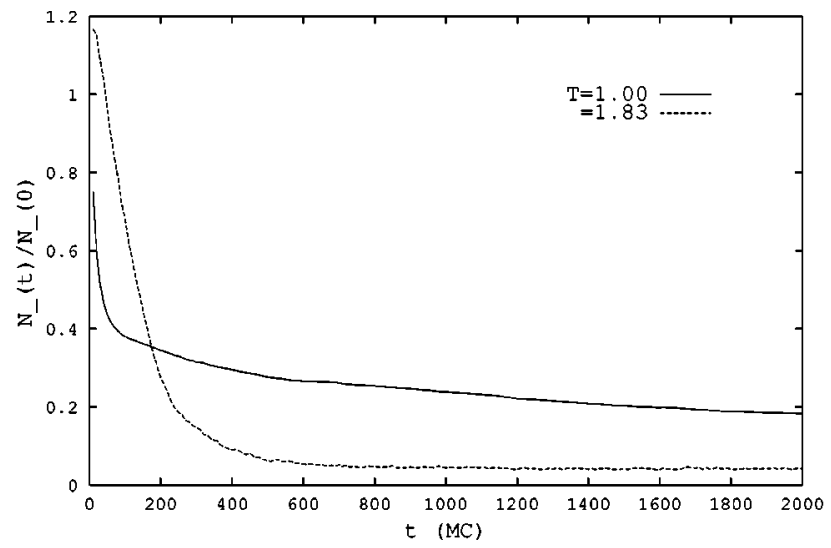

(a)

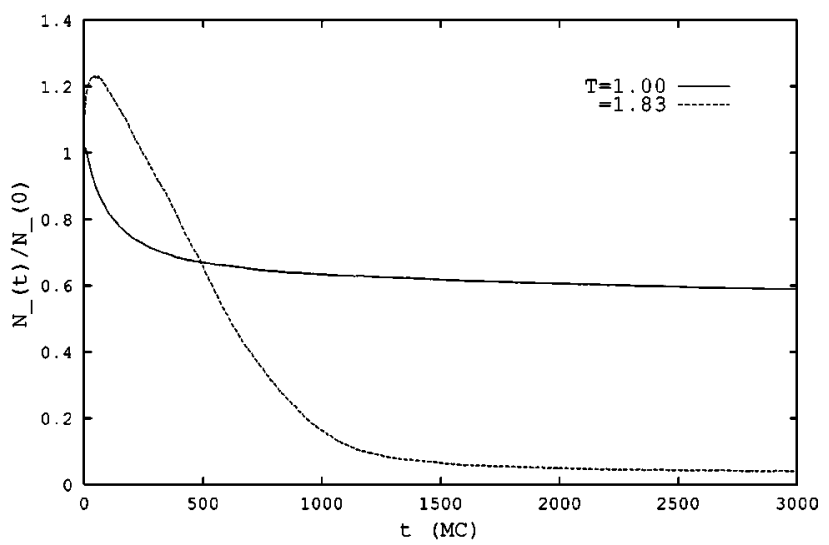

(b)

FIG. 2. The relaxation for two diferent temperatures $T=1.0$ and $T=1.83$ : (a) $16^{3}$ lattice volume with an initial chessboardlike configuration in a $8^{3}$ volume; (b) $30^{3}$ lattice volume with an initial chessboardlike configuration in a $20^{3}$ volume.

ferromagnetic ground state. We use fixed boundary conditions in order to make the system decay to a ferromagnetic ground state. Our measured quantity is the number of the minus spins, $N_{-}(t)$, in terms of the computing time divided by the number of spins at time $t=0, N_{-}(0)$. The quantity $N_{-}(t)$ is clearly related to the magnetization $M$, since $M$ $\approx N_{+}+N_{-}=N_{\text {tot }}-2 N_{-}$. Two examples of our results are given in Figs. 2(a) and 2(b) what have been produced from simulations over two lattice volumes, namely, $16^{3}$ and $30^{3}$ which at time $t=0$ have enclosed $8^{3}$ and $20^{3}$ lattice volumes, respectively, with a chessboardlike arrangement for the spins.

The two curves in each panel have been generated after averaging over a sample of 50-100 copies where each evolves starting from the same initial configuration, in order to reduce the noise. Two cases are shown. One is for temperature $T=1.83$, in the supercooled phase, while the other one, $T=1.0$, lies in the glassy phase. The difference on the relaxation time between the two temperatures is obvious. For the $T=1.83$ case the system reaches a stable value relatively fast for both lattice volumes, but this is not the case for $T$ $=1.0$. Note that the slope of the curve keeps taking a nonzero value even for remarkably large times. This is clear in

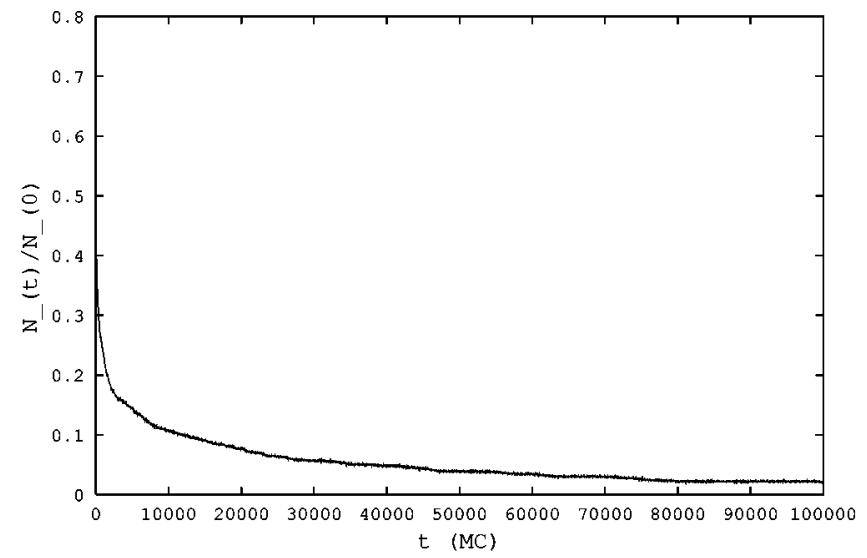

(a)
Fig. 3 where long runs are depicted for $T=1.0$, confirming that we are in the presence of slow dynamics. This behavior can be seen in a more apparent way in Fig. 4 where the results of Fig. 3 are presented in a logarithmic time scale. In Fig. 4 the logarithmic decay is present clearly enough.

The very distinct dynamics between the glassy phase and the evolution of the supercooled phase in the metastability region $T_{g}<T<T_{c}$ can be seen in a more definite way in Fig. 5. In this plot, the results for four temperatures are presented for the bigger volume used i.e., $30^{3}$. All of them correspond to a random starting configuration. For $T=1.83$, which lies in the metastability region, the evolution seems very fast all the way to the equilibrium value. On the contrary for the other three temperatures, a fast evolution is initially observed followed by a very slow one that persists up to very long times.

These results are very suggestive and indeed show that the system finds it very difficult to overcome dynamical energy barriers that are created along the evolution and this is undoubtedly the reason for the slow dynamics. Recall that the energy of the model is concentrated on the edges; the system has vanishing microscopic surface tension. To reduce the volume of the excitation with local moves, the total edge

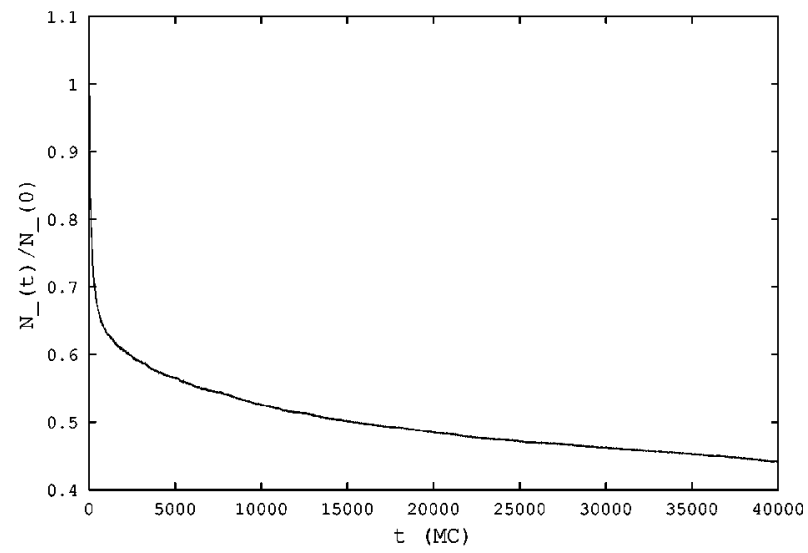

(b)

FIG. 3. The long-time evolution at $T=1.0$ for inner chessboardlike configuration in a $8^{3}$ (a) and $20^{3}$ volume (b). 


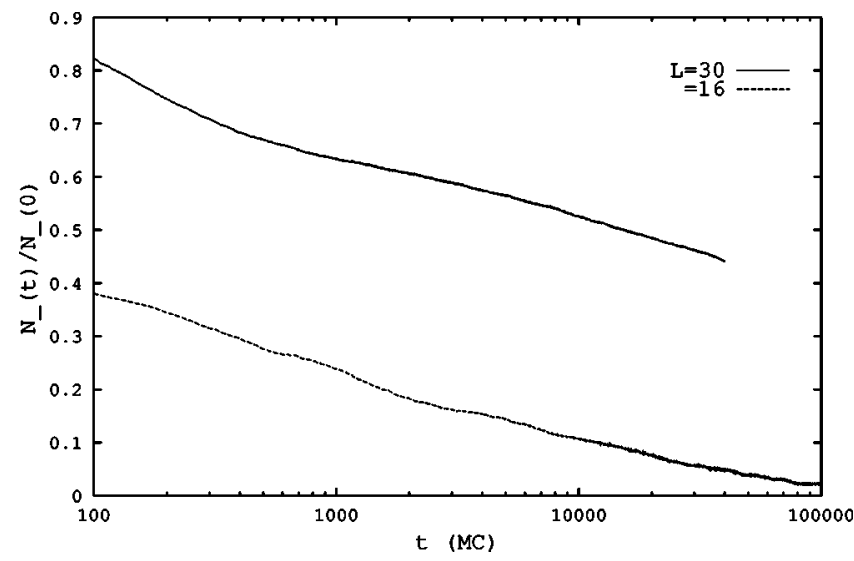

FIG. 4. The Fig. 3 results as plotted in a logarithmic time scale. $\left(8^{3}\right.$ and $20^{3}$ initial chessboardlike configurations for total volumes $16^{3}$ and $30^{3}$, respectively.)

length must temporarily increase by a substantial amount. That makes excitations such as the one we have been analyzing virtually stable.

In spite of the unambiguity of the previous results, in order to determine some properties associated to the very slow dynamics observed at low temperatures, we shall proceed to analyzing several dynamical correlators.

We shall first study the spin-spin autocorrelation function for temperatures lying in the metastability region after a random start, thus forcing the system to be in a supercooled phase. Its definition is given by

$$
C(t, w)=\frac{1}{N}\left\langle\sum_{\vec{r}} \sigma_{\vec{r}}(w) \sigma_{\vec{r}}(t+w)\right\rangle .
$$

The brackets mean that we take the average value over copies starting from a random configuration (200-400 in our case). The waiting time $w$ is the time for which the system is being thermalized before taking the measurements at subsequent times denoted by $t$. The waiting time $w$ is taken to be about 300 by noticing that, from that value on the resultant

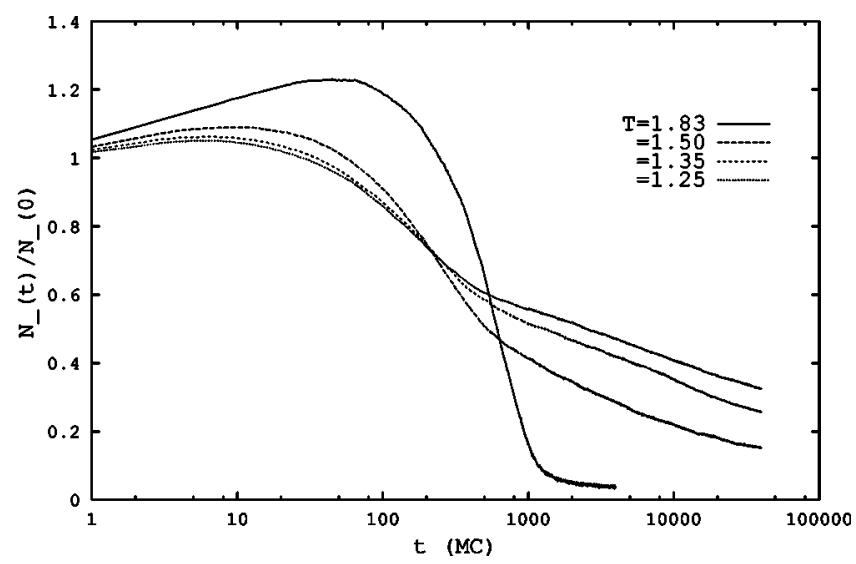

FIG. 5. For $T=1.83$ the system with lattice volume $V=30^{3}$ is in the supercooled region and exhibits fast relaxation. For the three other temperature values that tie in the cold phase the slow decay is obvious when it is presented in logarithmic time scale.

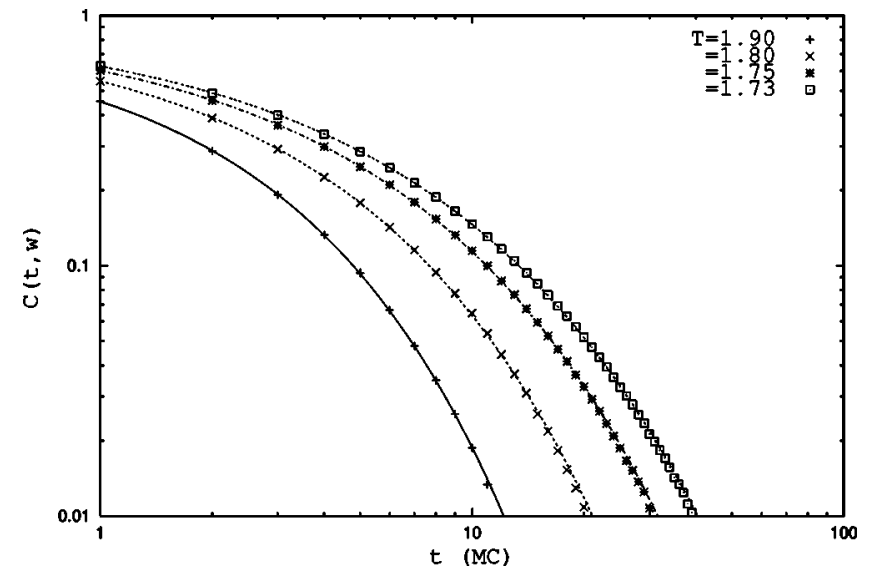

FIG. 6. Spin-spin autocorrelation function versus time for $V$ $=40^{3}$. The fittings to the points are of the stretched exponential type.

values for $C$ are quite identical as long as the temperature lies in the metastability region. In Fig. 6 we present some of our results for $C$ in a $40^{3}$ lattice volume and for four temperature values. The fittings are stretched exponentials of the form $a e^{-(t / \tau)^{b}}$. We denote by $\tau$ the relaxation time. In all cases we found $0.60<b<0.80$ with an error smaller than 0.004 . Since the fits seem quite good we go on and make a plot of the resulting values of $\tau$ versus the temperature $T$. The result is shown in Fig. 7 where the corresponding fit to a function having the form const/ $\left(T-T_{g}\right)^{c}$ is quite good and leads to the prediction $T_{g}=1.698$ (1) with $c=0.41$. At $T_{g}$ the autocorrelation time is expected to diverge because of the onset of the slow dynamics that turns the stretched exponential behavior into a power law (with a small exponent) or a logarithm.

Although that result is in good agreement with previous simulations $[12,14]$ the method relying on the stretched exponential fit may prove to be too risky for an exact prediction of $T_{g}$ due to ambiguities in the fitting process and perhaps is not trustworthy to that accuracy. As an alternative and a cross check, for two different temperature values, namely $T$ $=1.720$ and $T=1.695$ we show the behavior of the $C$ for two

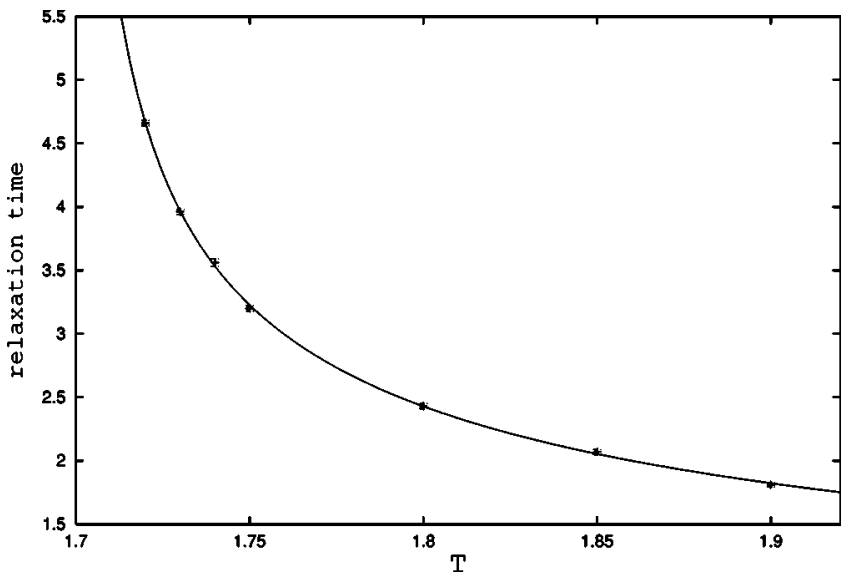

FIG. 7. Relaxation time $\tau$ versus $T$. The fitting procedure leads to divergent $\tau$ for $T_{g}=1.698$. 


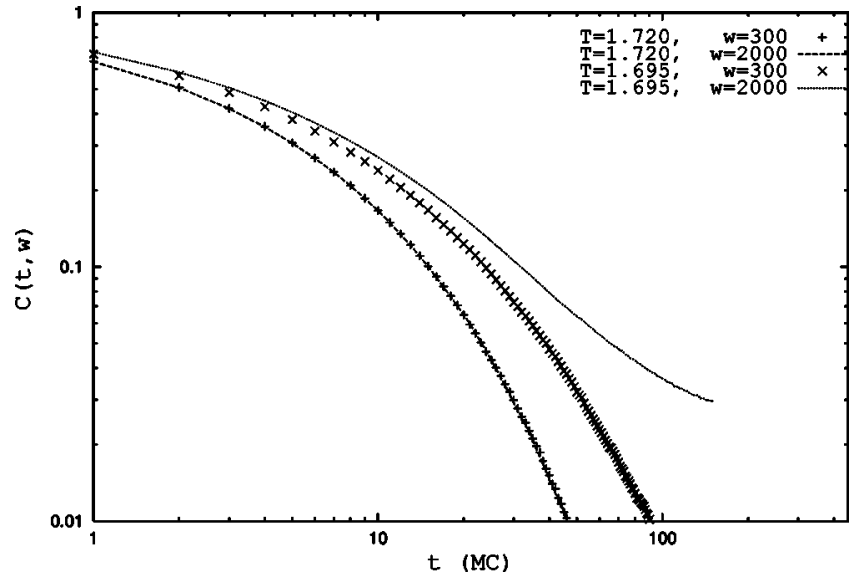

FIG. 8. $C$ versus time for two values for the waiting time $w$ in volume $V=40^{3}$. For the system in the glassy phase we see the dependence on $w$.

quite different values of the waiting time, $w=300,2000$. One sees that for the higher temperature the behavior of the $C$ is identical, but for the smaller one there is a strong dependence on $w$ showing that for larger waiting times the system exhibits different and, in particular, slow dynamics behavior which is a strong signal that the system has passed to the glassy phase. Hence we are able to estimate the value of $T_{g}$ to lie in the interval $1.695<T_{g}<1.720$, which indeed agrees also with the previously obtained value, (see Fig. 8).

\section{NEXT AND NEXT-TO-NEAREST NEIGHBOR INTERACTION $(\kappa=1)$}

As we have indicated the gonihedric spin model is actually a family of models parametrized by a real quantity $\kappa$. All the members of this family share the common feature of having their spin interfaces weighed with the total edge length. The value of $\kappa$ simply indicates the degree of selfavoidance of the surfaces. The plaquette model whose dynamical properties we just discussed corresponds to $\kappa=0$. An interesting member of this family is given by $\kappa=1$. The degeneracy of the ground state in this case is $3 \times 2^{L}$. Other values of $\kappa$ (in particular closer to the presumed tricritical point where the transition turns first order) were tentatively investigated in Ref. [3]. In view of the results of the present work, these preliminary investigations require a more detailed analysis.

In the present case the Hamiltonian is given by

$$
H=-2 \sum_{\vec{r}, \vec{\alpha}} \sigma_{\vec{r}} \sigma_{\vec{r}+\vec{\alpha}}+\frac{1}{2} \sum_{\vec{r}, \vec{\alpha}, \vec{\beta}} \sigma_{\vec{r}} \sigma_{\vec{r}+\vec{\alpha}+\vec{\beta}}
$$

As we see, the spin plaquette term has disappeared. From a practical point of view this model may be particularly interesting as the plaquette term is obviously hard to get in real materials. From the standpoint of a spin system this is just a model with nearest and next-to-nearest neighbor interactions (though a finely tuned one). As it has already been mentioned in the Introduction, the gonihedric model with $\kappa=1$ lies just outside the boundaries of the family of models considered in

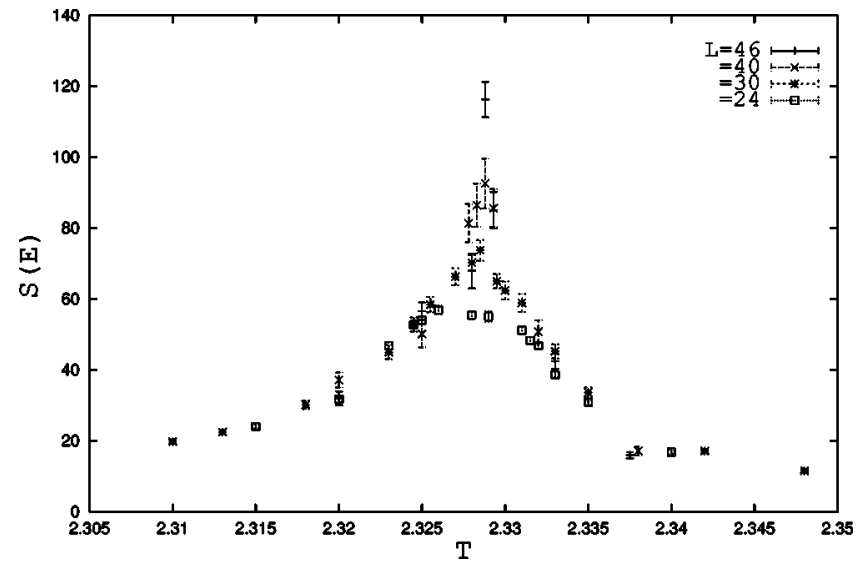

FIG. 9. $S(E)$ versus $T$ behavior with the lattice volume.

Ref. [8]. It also exhibits a large degeneration of the ground state, which is absent in Ref. [8].

We shall proceed to study the dynamical properties of the system by using Monte-Carlo methods. In performing the simulations we used the METROPOLIS algorithm for several lattice volumes, namely $10^{3}, 16^{3}, 20^{3}, 24^{3}, 30^{3}, 40^{3}$ and $46^{3}$. We imposed either fixed or periodic boundary conditions depending on the kind of the measurement that was performed. During the presentation of our results, the conditions we used will be explicitly mentioned.

We begin by giving the behavior for the susceptibility of the energy with the lattice volume which is given by

$$
S(E)=V\left(\left\langle E^{2}\right\rangle-\left\langle E^{2}\right\rangle\right)
$$

We denote by $V$ the lattice volume and by the symbol \langle\rangle , the average value over sweeps. In Fig. 9 the susceptibility for the system energy as a function of the temperature for four lattice volumes is depicted. For every point in the figure we performed $10^{5}$ thermalization sweeps followed by more than $10^{5}$ measurements, using periodic boundary conditions and starting from the ordered configuration. The peak for each volume clearly increases with it although with an exponent less than one, which is a signal for a second-order phase transition. Also, the positions of the peaks for the bigger volumes are seen to concentrate around the value $T_{c}$ $=2.329$ which is the pseudocritical value for the volumes we used. It should be mentioned that while the second order character of the phase transition was already mentioned in Refs. [6,18], our prediction for $T_{c}$ gives a somewhat smaller value.

Having estimated the critical temperature value for the system, we want now to study the dynamic behavior at low temperatures, i.e., below $T_{c}$. To this end, we use first the same method as for the $\kappa=0$ case, described in the previous section, which consists of choosing an initial chessboardlike configuration defined in a cubic subvolume of the system. We impose fixed boundary conditions and study the behavior of the dynamical quantity

$$
e(t)=\frac{E(t)-E_{e q}}{E(0)-E_{e q}}
$$




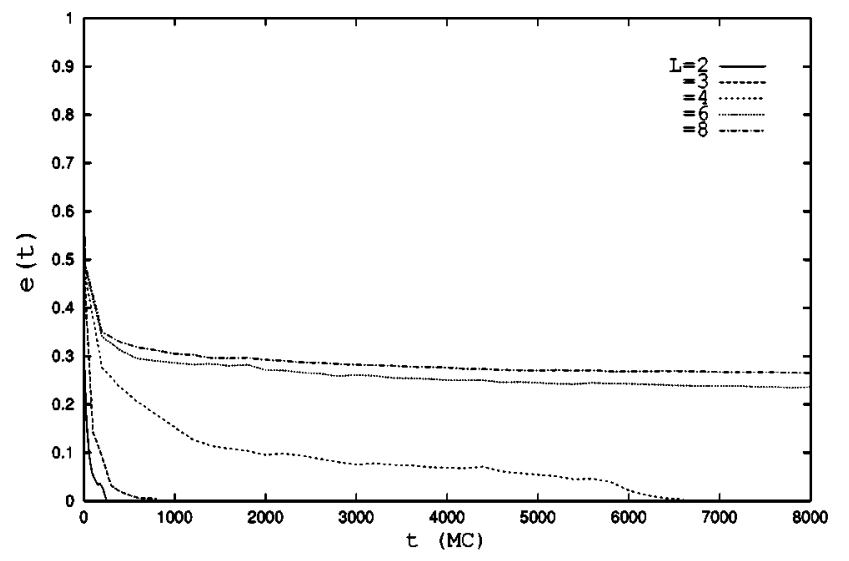

(a)

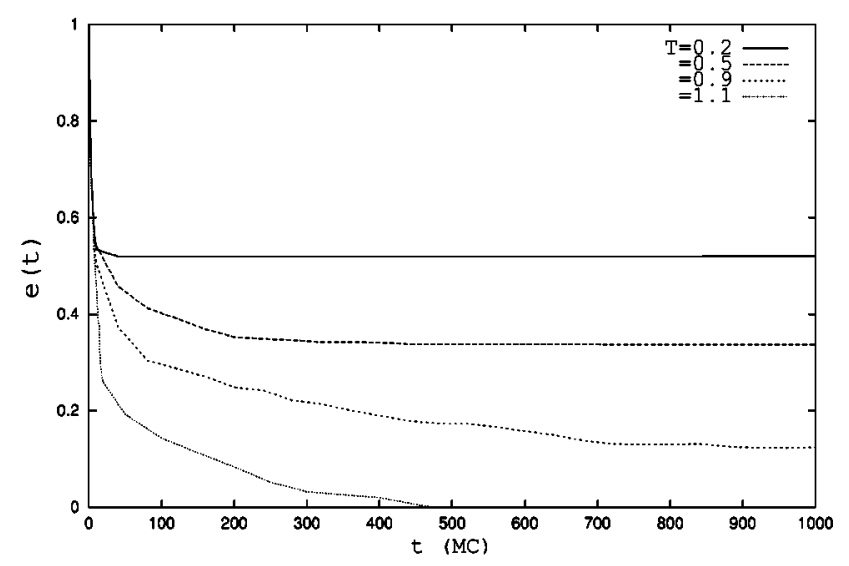

(b)

FIG. 10. The relaxation behavior of the initial configuration as seen by the study of quantity defined in Eq. (5) in lattice volume $V$ $=16^{3}$ : (a) for various inner volume sizes at fixed temperature, $T=1.1$ (from top to bottom $L=2,3,4,6,8$ ) and (b) for fixed inner volume size ( $L=3$ ) with decreasing temperature (from top to bottom $T=0.2,0.5,0.9,1.1$ ).

with time for various temperatures in the cold phase and for different sizes of the inner chessboard-like volume. In Eq. (5), $E(t)$ and $E(0)$ denote the energy of the system at time $t$ and just after the first sweep is performed, respectively, and $E_{e q}$ is the system energy when an ordered configuration is taken as the initial one. In Fig. 10(a) we give an example for lattice volume $16^{3}$. We fix the temperature at the value $T$ $=1.1$ and we are interested in the behavior as the dimension of the inner chessboard-like volume increases from $L=2$ to $L=8$. Each curve has been produced after averaging several hundreds of repetitions starting from the same initial configuration in order for the noise to be reduced. The results show a dramatic increase in the relaxation time with increasing system size. For the maximum value of $L$ used, the observable (5) shows very slow variation with the time and it follows a power law behavior with estimated exponent equal to 0.18 , so a logarithmic behavior cannot be really excluded.

We can reach similar conclusions by studying the above relaxation quantity for decreasing values of temperature at fixed $L=3$. In Fig. 10(b) the corresponding results are depicted for a $16^{3}$ total volume. In particular for the value $T$

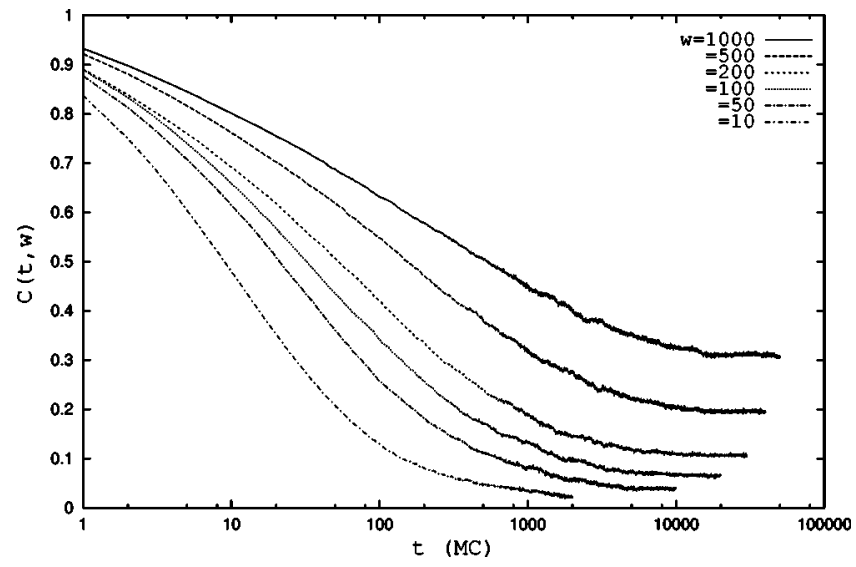

FIG. 11. The spin-spin autocorrelation function in $V=24^{3}$ and for $T=2.2$ as depicted for different waiting times $w$ (from top to bottom $w=1000,500,200,100,50,10)$.
$=0.2$, the dynamics is so slow that it seems really hard for the system to get to the ground state for accessible computing times.

Next we shall move to a more quantitative study following the same lines as in the preceding section. We look for aging features by considering the autocorrelation spin-spin function defined by Eq. (3). Brackets indicate that we take the average value over 100-200 trials starting from a random configuration. $w$ is the time for which the system is being thermalized before taking the measurements at subsequent times denoted by $t$. We follow the same lines of analysis as in Ref. [14] which concerns the $\kappa=0$ case. In Fig. 11 we show an example of the behavior of the autocorrelation function (3) for the temperature value $T=2.2$ and for a $24^{3}$ lattice volume. Six different curves for the $C$ are depicted, each corresponding to a different value of $w$. The very slow evolution of the autocorrelation function combined with the clear dependence on the time $w$ give strong evidence in favor of aging. In order to determine the aging (whether I or II) we consider the overlap function [19]

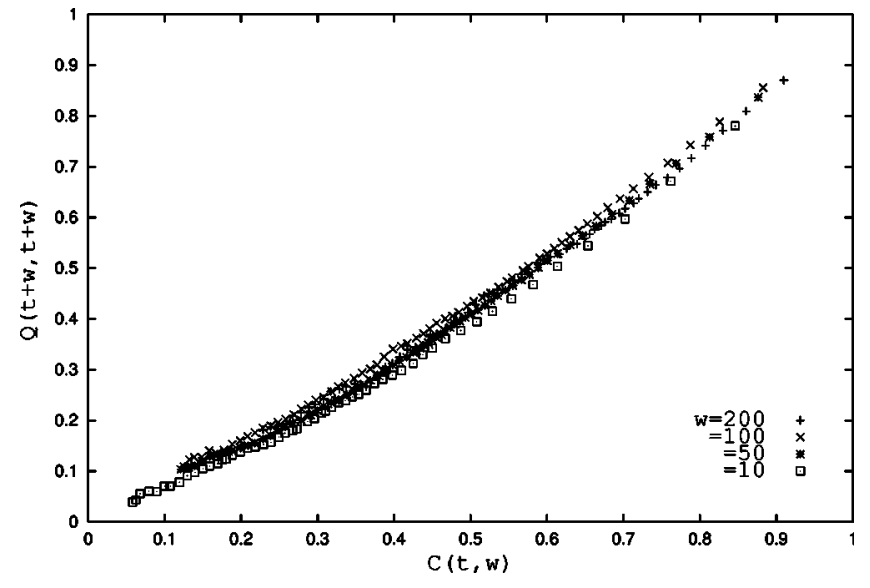

FIG. 12. The $Q(t+w, t+w) \rightarrow 0$ behavior as $C(t, w) \rightarrow 0$ is clearly seen for the four values of $w\left(V=24^{3}\right.$ and $\left.T=2.2\right)$. 


$$
Q(t+w, t+w)=\frac{1}{N}\left\langle\sum_{\vec{r}} \sigma_{\vec{r}}^{(1)}(t+w) \sigma_{\vec{r}}^{(2)}(t+w)\right\rangle .
$$

The measuring process for the above consists of the relaxation of the system for time $w$ starting from the random configuration. At that moment we take two copies $\sigma^{(1)}$ and $\sigma^{(2)}$ each evolving independently for time $t$. In Fig. 12 we show the behavior of $Q(t+w, t+w)$ in terms of $C(t, w)$ for the same values of the volume and temperatute as in Fig. 11 and for four values of the waiting time $w$. It clearly follows that as $C(t, w) \rightarrow 0, Q(t+w, t+w) \rightarrow 0$. The fact that the two system copies are moving independently since they show zero overlapping indicates strong evidence for type-II aging.

\section{CONCLUSIONS}

At this point we would like to summarize our main conclusions. In this work we have carried out a rather complete analysis of the dynamical properties of the gonihedric spin model in three dimensions. This model has a clear geometrical inspiration derived from its simplicity and connection to random surface theory. A self-avoidance parameter $\kappa$ parametrizes different models in this family. Here we have explicitly considered $\kappa=0$ and $\kappa=1$.

The model exhibits large potential barriers that are dynamically generated along the evolution process while trying to adjust to the environment. This is due to the fact that the area term of the interfaces is completely subleading and the dynamics is driven by the total edge length of the excitations. This creates basins of stability, from where it is virtually impossible to kick out the system by local thermal fluctuations. This happens both for $\kappa=0$ (a purely plaquette interaction) and $\kappa=1$ (no plaquette interaction and only competing nearest and next-to-nearest neighbor interactions). This latter case lies just outside the boundaries of models of competing ferromagnetic nearest interactions and antiferromagnetic next-to-nearest neighbor interactions previously considered. We believe a large amount of additional symmetry is present in the gonihedric limiting case.

Several algorithms have been used to obtain results: Glauber dynamics, heat bath and standard METROPOLIS. Modulo an overall rescaling of Monte-Carlo time, the results are fully equivalent.

For the $\kappa=0$ case we give evidence of a glassy transition and we estimate the temperature value $T_{g}$ at which the glassy phase arises being in the interval $[1.695,1.720]$. We show that the dynamics is almost certainly logarithmic for $T$ $<T_{g}$. In the mestatability region, above $T_{g}$ but below the true thermodynamical transition $T_{c}$ (where the system has a first-order transition) the approach to equilibrium is well described by a stretched exponential, whose characteristic time scale diverges as $T \rightarrow T_{g}^{+}$.

We confirm that for the case $\kappa=1$ the system exhibits a second-order phase transition. The critical properties of this transition have not been elucidated yet; a tentative determination of the exponents using finite size scaling carried out in Ref. [3] gives a rather nonstandard set of values. Work on this is in progress, but the problem is aggravated by the presence of slow dynamics. Indeed we have detected very slow dynamical behavior, quite similar to that takes place with $\kappa=0$ for $T<T_{g}$ in the cold phase of this system. This dynamical behavior has all features of the type-II aging and is again compatible with logarithmic evolution. From our results we conclude that it extends all the way up to $T_{c}$.

It is interesting to speculate about the role played by the flipping symmetry present in the case $\kappa=0$. As has been mentioned in the Introduction, this symmetry may make the evolution rather nonobvious (it is not difficult to come up with configurations where, due to the symmetry, there are subtantial domain growths at no energy cost). However, in a local algorith (such as GLAUBER or METROPOLIS) this symmetry is presumably largely irrelevant. Indeed, to flip one plane one has to flip each spin in it, one after the other. While in a "normal" spin system this possibility would be suppressed by $\sim e^{-L^{2}}, L$ being the linear size of the system, in the gonihedric model the suppression would be much smaller, just $\sim e^{-L}$. We believe that this is still too small to play a significant role in the coarsening process after a sudden quench, for instance, since the typical size of the domains will be much smaller than the linear size $L$. But undoubtedly, it should play a crucial role in determining equilibrium properties of the system.

Possible applications of these simple, but interesting, spin models are discussed.

\section{ACKNOWLEDGMENTS}

D.E. thanks D. Johnston and A. Lipowski for discussions and the hospitality of the Department of Mathematics of Heriot-Watt University. P.D. is grateful to G. Koutsoumbas for useful discussions and acknowledges support from "EUROGRID-Discrete random geometries: from solid state physics to quantum gravity" (Grant No. HPRN-CT-199900161). The support from Grant Nos. MCyT FPA 2001-3598 and CIRIT 200156R-00065 is also acknowledged. Part of this work was carried out at CESCA.
[1] R.V. Ambartzumian, G.S. Sukasian, G.K. Savvidy, and K.G. Savvidy, Phys. Lett. B 275, 99 (1992); G.K. Savvidy and K.G. Savvidy, Int. J. Mod. Phys. A 8, 3393 (1993); G.K. Savvidy and K.G. Savvidy, Mod. Phys. Lett. A 8, 2963 (1993).

[2] G.K. Savvidy and F.J. Wegner, Nucl. Phys. B 413, 605 (1994); G.K. Savvidy and K.G. Savvidy, Phys. Lett. B 324, 72 (1994); G.K. Savvidy, K.G. Savvidy, and F.J. Wegner, Nucl. Phys. B 443, 565 (1995); J. Ambjorn, G. Koutsoumbas, and G.K. Sav- vidy, Europhys. Lett. 46, 319 (1999).

[3] M. Baig, D. Espriu, D. Johnston, and R.P.K.C. Malmini, J. Phys. A 30, 7695 (1997).

[4] D. Alvarez, S. Franz, and F. Ritort, Phys. Rev. B 54, 9756 (1996).

[5] G. Koutsoumbas, G.K. Savvidy, and K.G. Savvidy, Phys. Lett. B 410, 241 (1997).

[6] G.K. Bathas, E. Floratos, G.K. Savvidy, and K.G. Savvidy, 
Mod. Phys. Lett. A 10, 2695 (1995).

[7] G.K. Savvidy, J. High Energy Phys. 9, 044 (2000).

[8] J.D. Shore, M. Holzer, and J.P. Sethna, Phys. Rev. B 46, 11376 (1992)

[9] D. Espriu, M. Baig, D.A. Johnston, and R.K.P.C. Malmini, J. Phys. A 30, 405 (1997).

[10] G. Koutsoumbas and G.K. Savvidy, e-print cond-mat/0111590.

[11] E.N.M. Cirillo, G. Gonnella, D.A. Johnston, and A. Pelizzola, Phys. Lett. A 226, 59 (1997).

[12] A. Lipowski, J. Phys. A 30, 7365 (1997).

[13] A. Lipowski and D. Johnston, e-print cond-mat/9812098.

[14] M.R. Swift, H. Bokil, R.D.M. Travasso, and A.J. Bray, Phys. Rev. B 62, 11494 (2000).
[15] A. Lipowski and D. Johnston, Phys. Rev. E 61, 6375 (2000).

[16] A. Lipowski and D. Johnston, Phys. Rev. E 64, 041605 (2001).

[17] A. Lipowski, D. Johnston, and D. Espriu, Phys. Rev. E 62, 3404 (2000).

[18] D.A. Johnston and R.P.K. Malmini, Phys. Lett. B 378, 87 (1996).

[19] A. Barrat, R. Burioni, and M. Mezard, J. Phys. A 29, 1311 (1996).

[20] G.K. Savvidy, e-print cond-mat/0003220.

[21] R.J. Glauber, J. Math. Phys. 4, 294 (1963).

[22] G.K. Savvidy, K.G. Savvidy, and P.G. Savvidy, Phys. Lett. A 221, 233 (1996). 